\title{
Stability and antimicrobial effect of amikacin-loaded solid lipid nanoparticles
}

\author{
This article was published in the following Dove Press journal: \\ International Journal of Nanomedicine \\ 15 December 2010 \\ Number of times this article has been viewed
}

\author{
Solmaz Ghaffari' \\ Jaleh Varshosaz' \\ Afrooz Saadat ${ }^{2}$ \\ Fatemeh Atyabi \\ 'Department of Pharmaceutics, \\ Faculty of Pharmacy and Isfahan \\ Pharmaceutical Sciences Research \\ Center, Isfahan University of Medical \\ Sciences, Isfahan, Iran; ${ }^{2}$ Department of \\ Pharmaceutics, Faculty of Pharmacy, \\ Tehran University of Medical Sciences, \\ Tehran, Iran
}

Correspondence: Jaleh Varshosaz Department of Pharmaceutics, Faculty of Pharmacy and Isfahan Pharmaceutical Sciences Research Center, Isfahan University of Medical Sciences, Isfahan, Iran

Tel +98 3 I I 7922579

Fax +983116680011

Email varshosaz@pharm.mui.ac.ir

\begin{abstract}
Solid lipid nanoparticles (SLNs) of amikacin were designed in this study for pulmonary delivery to reduce the dose or its administration intervals leading to reduction of its toxicities especially in long term treatment. Nanoparticles of amikacin were prepared from cholesterol by solvent diffusion technique and homogenization. The size, zeta potential, loading efficiency, and release profile of the nanoparticles were studied. The conventional broth macrodilution tube method was used to determine the minimum inhibitory concentration (MIC) and minimum bacteriostatic concentration (MBC) of amikacin SLNs with respect to Pseudomonas aeruginosa in vitro. To guarantee the stability of desired SLNs, they were lyophilized using cryoprotectants. Results showed that considering the release profile of amikacin from the studied nanocarrier, MIC and MBC of amikacin could be about two times less in SLNs of amikacin compared to the free drug. Therefore, fewer doses of amikacin in SLNs can clear the infection with less adverse effects and more safety. Particle size enlargement after lyophilization of desired SLNs after two months storage was limited in comparison with non-lyophilized particles, 996 and $194 \mathrm{~nm}$, respectively. Zeta potential of lyophilized particles was increased to $+17 \mathrm{mV}$ from $+4 \mathrm{mV}$ before lyophilization. Storage of particles in higher temperature caused accelerated drug release.
\end{abstract}

Keywords: amikacin, antimicrobial effects, Pseudomonas aeruginosa, solid lipid nanoparticles, stability

\section{Introduction}

Aminoglycosides are the mainstay in the treatment of serious Gram-negative systemic infections. The use of aminoglycosides can be limited because of their adverse effects, mainly oto- and nephrotoxicity which happens in 15\%-17\% of patients, hearing loss $(8 \%)$, and vestibular toxicity $(3 \%)$. Also retina toxicity was observed with gentamycin in vitro. ${ }^{1-3}$ Controlling aminoglycosides concentration is critical because of their narrow therapeutic range. ${ }^{3}$ Aminoglycosides are used for treatment of many infections, such as severe pulmonary infections like cystic fibrosis and skin infections, because of their beneficial effects. ${ }^{4}$ Many studies have been carried out so far to reduce these toxicities such as production of liposomal amikacin dry powder inhaler, intra-tracheal delivery strategy of gentamycin, and thiolated chitosan nanoparticles of amikacin. ${ }^{5-7}$ Production of solid lipid nanoparticles (SLNs) of amikacin for pulmonary delivery was also reported by Varshosaz et al. ${ }^{8}$

There are some differences between liposomes and SLNs. By definition, a liposome is a tiny bubble (vesicle) made out of the same material as a cell membrane (lipid bilayer). Liposomes can be filled with drugs and be used to deliver drugs for 
cancer and other diseases. Membranes are usually made of phospholipids which are molecules that have a head group and a tail group. The head is hydrophilic and the tail, which is made of a long hydrocarbon chain, is hydrophobic. In nature, phospholipids are found in stable membranes composed of two layers (a bilayer). The heads are attracted to water and line up to form a surface facing the water, whereas the tails are repelled by water and line up to form a surface away from it. When membrane phospholipids are disrupted, they can reassemble themselves into tiny spheres, smaller than a normal cell, either as bilayers or monolayers. The bilayer structures are liposomes. The monolayer structures are called micelles. Liposomes can be composed of naturally-derived phospholipids with mixed lipid chains, like egg phosphatidylethanolamine, or of pure surfactant components, like dioleoylphosphatidylethanolamine. ${ }^{9}$ SLNs as colloidal carrier systems combine the advantages of traditional systems, but avoid some of their major disadvantages. In contrast to liposomes, SLNs do not have a bilayer structure. They may have a matrix structure or encapsulate drugs. They are much more stable than liposomes. Some advantages of SLNs are the possibility of controlling drug release and drug targeting, increased drug stability, high drug payload, possibility of the incorporation of lipophilic and hydrophilic drugs, lack of biotoxicity of the carrier, no problems with respect to large-scale production, sterilization possibility, and good tolerability. However, some of the major drawbacks of SLNs are low drug loading, unpredictable drug release, and the risk of gelation due to polymorphism of the solid lipids. The main ingredients used to produce SLNs include solid lipid(s), emulsifier, and water.

There are many studies on SLNs, even for hydrophilic drugs and peptide delivery. The desired SLNs were made from cholesterol with one layer structure, smaller particle size, simple preparation method, and good stability that candidates makes then for success in scale up in future. ${ }^{10,11}$

Amikacin is 6-O-(3-amino-3-deoxy- $\alpha$-1)-glucopyranosyl)4-O-(6-amino-6-deoxy- $\alpha$-D-glucopyranosyl)-N1-[(2,S)4-amino- 2-hydroxybutanoyl]-2-deoxy-D-streptamine, a substance obtained from kanamycin A. It has positive charge. Amikacin sulphate having a molar ratio of amikacin to $\mathrm{H}_{2} \mathrm{SO}_{4}$ of $1: 2$ contains the equivalent of not less than $674 \mu \mathrm{g}$ and not more than $786 \mu \mathrm{g}$ of amikacin $\left(\mathrm{C}_{22} \mathrm{H}_{43} \mathrm{~N}_{5} \mathrm{O}_{13}\right)$ per $\mathrm{mg}$, calculated on the dried basis. It has the following molecular formula $\mathrm{C}_{22} \mathrm{H}_{43} \mathrm{~N}_{5} \mathrm{O}_{13} 2 \mathrm{H}_{2} \mathrm{SO}_{4}$ with a molecular weight of 781.75. Aminoglycosides like amikacin "irreversibly" bind to specific $30 \mathrm{~S}$ subunit proteins and $16 \mathrm{~S}$ rRNA. Amikacin inhibits protein synthesis by binding to the $30 \mathrm{~S}$ ribosomal subunit to prevent the formation of an initiation complex with mRNA. Specifically amikacin binds to four nucleotides of $16 \mathrm{~S}$ rRNA and a single amino acid of protein S12. This interferes with the decoding site in the vicinity of nucleotide 1400 in $16 \mathrm{~S}$ rRNA of $30 \mathrm{~S}$ subunit. This region interacts with the wobble base in the anticodon of tRNA. This leads to interference with the initiation complex and misreading of mRNA so incorrect amino acids are inserted into the polypeptide leading to nonfunctional or toxic peptides and the breakup of polysomes into nonfunctional monosomes. Amikacin is a semisynthetic broad spectrum aminoglycoside antibiotic derived from kanamycin A by acetylation. It is commonly administered parenterally for the treatment of Gram-negative infections resistant to gentamycin, kanamycin, and tobramycin because the amikacin molecule has fewer points susceptible to enzymatic attack than the other aminoglycosides. ${ }^{4,9,12}$ Commonly used aminoglycosides include tobramycin, gentamycin, and amikacin. ${ }^{2}$ For treatment of cystic fibrosis, combination therapy with an aminoglycoside, a broad spectrum betalactam antibiotic is given intravenously for 14-21 days. ${ }^{4}$

The minimum inhibitory concentration (MIC) of amikacin is $8 \mu \mathrm{g} / \mathrm{mL}$ for Pseudomonas aeruginosa. ${ }^{1}$ In previous studies it has been reported that liposomal encapsulated tobramycin showed considerable antimicrobial effect at concentrations below the MIC of the free antibiotic in vitro. ${ }^{2,13}$ Therefore, reducing the effective concentration of aminoglycosides can be a challenge to reduce their adverse effects.

In the present study the minimum effect of SLNs of amikacin was investigated and compared with MIC of the free drug. The SLNs of amikacin were prepared for minimum size and maximum drug-loading efficiency using cholesterol as the dipodic ingredient. At first, the release profile of the drug from SLNs was studied, then a screening was performed to show the MIC of SLNs of amikacin.

To investigate the stability condition of desired nanoparticles, three different conditions were selected and desired SLNs were stored for two months and their physicochemical properties investigated for both particle dispersion and freeze-dried particles. The antimicrobial efficiency of both dispersion and dried SLNs was the same in first step of production.

\section{Material and methods Materials}

Cholesterol, Tween 80, ethanol, acetone, and Muller-Hinton agar were all from Merck Chemical Company (Darmstadt, Germany). P. aerogynosa (American Type Culture Collection 
[ATCC] 9027) was obtained from the microbiology laboratory in Tehran University of Medical Sciences (Tehran, Iran).

\section{Preparation of SLNs of amikacin}

The SLNs of amikacin were prepared as our previously reported $\operatorname{method}^{8}$ to achieve optimized particles from a particle size and drug-loading efficiency point of view. Briefly, $160 \mathrm{mg}$ of amikacin powder was dissolved in deionized water containing $1 \% \mathrm{w} / \mathrm{w}$ Tween 80 and homogenized at 11,000 rpm ( $\mathrm{T} 18$ basic Ultra-Turrax; IKA Werke $\mathrm{GmbH}$, Staufen, Germany). Then $314 \mathrm{mg}$ of cholesterol $(0.81 \mathrm{mmol})$ as lipid phase was dissolved in $24 \mathrm{~mL}$ of the mixture of ethanol/acetone with the ratio of $3: 1(\mathrm{v} / \mathrm{v})$ (equal to $18 \mathrm{~mL}$ ethanol and $6 \mathrm{~mL}$ acetone) by heating to $70^{\circ} \mathrm{C}$ and stirring. Then hot oily phase was added to aqueous phase in $25^{\circ} \mathrm{C}$ under homogenization at $11,000 \mathrm{rpm}$ for $6 \mathrm{~min}$. The prepared emulsion was sonicated in bath sonicator (Tecno-Gaz Ultrasonic system; Tecna S.p.A, Bologna, Italy) and cooled to room temperature to achieve nanoparticles. SLNs were produced in optimum level and type of surfactant, optimum rate and time of homogenization, and ratio of lipid:drug to achieve smallest particles with maximum drug-loading efficiency using central composite design. ${ }^{8}$

Particle size and zeta potential of produced SLNs were measured using photon correlation spectroscopy (Zetasizer Nano ZS 3000; Malvern Instruments, Worcestershire, UK).

\section{Drug-loading efficiency}

Loading efficiency of amikacin in SLNs was calculated by Eq. $1{ }^{8,14}$ In this method the concentration of entrapped amikacin was calculated from the difference between the total amount of drug used for preparing SLNs and the free remaining drug in the medium. To do this, after preparing SLNs the emulsion containing SLNs was centrifuged (Sigma Laboratories, Osterode, Germany) at $35,000 \mathrm{rpm}$ for $45 \mathrm{~min}$ at $-4^{\circ} \mathrm{C}$ and concentration of amikacin in supernatant was analyzed by high-performance liquid chromatography (HPLC). ${ }^{8}$

$$
\underset{\text { Drug-loading }}{\text { efficiency }(\text { LE\% })}=\frac{\text { Drug }_{\text {total }}-\text { Drug }_{\text {supernatant }}}{\operatorname{Drug}_{\text {total }}} \times 100
$$

\section{Release study}

Release study was performed using dialysis method. Five $\mathrm{mL}$ of the optimized formulation before freeze-drying was placed in DO405 dialysis tubing $23 \times 15 \mathrm{~mm}$ (cut-off: $10-12 \mathrm{KD}$; Sigma Laboratories, Osterode, Germany) immersed in $50 \mathrm{~mL}$ of phosphate buffer solution ( $\mathrm{pH}$ 7.4). One $\mathrm{mL}$ sample was withdrawn at predetermined time intervals and drug concentration was analyzed using pre-column derivatization by HPLC method. ${ }^{8}$ Drug release profile was studied in different temperature conditions for SLNs of amikacin solution in first dispersion and redispersed freeze-dried nanoparticles after storage at $4{ }^{\circ} \mathrm{C}, 25^{\circ} \mathrm{C}$, and $40^{\circ} \mathrm{C}$ for 60 days.

\section{Freeze drying}

Lyophilization was used to prolong stability of amikacin loaded SLNs. Lyophilization of particles was done using cryoprotectants like sucrose, dextrose, and mannitol to limit the risk of aggregation of particles. The process was optimized using D-optimal experimental design reported previously. ${ }^{15}$

\section{Stability study}

The initial particle size and zeta potential of the amikacin loaded nanoparticles dispersion were measured immediately after preparation and after freeze drying, and redispersion of particles in deionized water by bath sonication for $5 \mathrm{~s}$ using Zetasizer Nano ZS3000 (Malvern, UK). This batch was divided into three sample sets and each sample set was stored at $4^{\circ} \mathrm{C}, 25^{\circ} \mathrm{C}$, and $40^{\circ} \mathrm{C}-4^{\circ} \mathrm{C}$ being refrigerator conditions, $25^{\circ} \mathrm{C}$ for room temperature, and $40^{\circ} \mathrm{C}$ as the indicative hot climate areas that the drug may be stored during distribution from the manufacturer to the end user.

All samples were stored in plain glass vials (USP type 1). Samples were withdrawn after 1, 3, 7, 15, 30, 45, and 60 days and subjected to particle size and zeta potential measurements. Polydispersity index (PDI) and drug released in deionized water containing $1 \% \mathrm{w} / \mathrm{w}$ Tween 80 were studied. However, measurement of released fraction of drug was checked just after 60 days as the samples were dry and had no risk of drug release. ${ }^{16}$

\section{Morphology study}

Morphology of the nanoparticles was characterized by scanning electron microscopy (SEM). The nanoparticles were mounted on aluminum stubs, sputter-coated with a thin layer of $\mathrm{Au} / \mathrm{Pd}$, and examined using an SEM (Philips XL30; Philips, Almelo, The Netherlands).

\section{Antimicrobial activity of SLNs}

To determine if there is any relationship between the activity of SLNs of amikacin and drug release profile from colloidal vehicle, and also to compare between the activity of nanoparticles of amikacin (directly after preparing particles in original medium and after lyophilization of SLNs and then 
dispersed in water) with that of free drug, the "well diffusion test" was carried out using P. aeruginosa (ATCC 9027) as the Gram-negative pathogenic strain.

The bacterial suspensions with a cell density equivalent to $0.5 \mathrm{McF}$ arland $\left(1.5 \times 10^{8} \mathrm{CFU} / \mathrm{mL}\right)$ were transferred individually onto the surface of Muller-Hinton agar plates using sterile cotton swabs. Wells with $8 \mathrm{~mm}$ diameters were prepared by punching a sterile cork borer onto agar plates and removing the agar to form a well. Aliquots of $100 \mu \mathrm{l}$ of each of two control solutions, free-drug and blank-SLNs, were delivered into the wells. A third well was full with SLNs of amikacin as the test sample. After incubation time for about $24-48 \mathrm{~h}$, at $35^{\circ} \mathrm{C}-37^{\circ} \mathrm{C}$, the zones of inhibition around the wells were measured in mm using a caliper.

\section{Determination of MIC and minimum bacteriostatic concentration of SLNs against the pathogen}

The conventional broth macrodilution tube method was used to determine MIC and minimum bacteriostatic concentration (MBC) of SLNs of amikacin with respect to $P$. aeruginosa as Gram-negative pathogenic bacteria in vitro. ${ }^{17,18}$ A stock solution of free amikacin was prepared in sterile water $(64 \mu \mathrm{g} / \mathrm{mL})$ that was further diluted in Muller-Hinton broth to reach a concentration range of 0.125 to $32 \mu \mathrm{g} / \mathrm{mL}$. SLNs were also dispersed in Muller-Hinton broth to reach an equal concentration of free amikacin as 0.125 to $32 \mu \mathrm{g} / \mathrm{mL}$ according to the percent of drug loading.

Final concentration of bacteria in individual tubes was adjusted to about $5 \times 10^{6} \mathrm{CFU} / \mathrm{mL}$. Control tubes contained only culture media without any antimicrobial agent, culture media with SLNs of amikacin due to probable contamination. Other test tubes included culture media with pathogenic strain (in $5 \times 10^{6} \mathrm{CFU} / \mathrm{mL}$ ), culture media with amikacin SLNs and pathogenic strain (in $5 \times 10^{6} \mathrm{CFU} / \mathrm{mL}$ ), and culture media with $P$. aeruginosa (in $5 \times 10^{6} \mathrm{CFU} / \mathrm{mL}$ ) with free amikacin in MIC $(8 \mu \mathrm{g} / \mathrm{mL})$, as was previously measured. ${ }^{1,2}$

After 24 and $48 \mathrm{~h}$ incubation at $35^{\circ} \mathrm{C}-37^{\circ} \mathrm{C}$, the test tubes were examined for possible bacterial turbidity and MBC and MIC of each test compound was determined respectively as lowest concentration that could stop and inhibit visible bacterial growth for $24 \mathrm{~h}$ and $72 \mathrm{~h} .{ }^{12,13}$ Figure 1 demonstrates the method of the MIC and MBC testing. A study was done on both freeze-dried and nonfreeze-dried SLNs of amikacin.

\section{Results and discussion Particle size}

The size and zeta potential of the particles before freeze drying were $150 \pm 4 \mathrm{~nm}$ and $+4 \mathrm{mV}$, respectively, and after freeze drying these increased to $190 \pm 7 \mathrm{~nm}$ and $+16 \mathrm{mV}$. The percentage of drug-loading efficiency was $87 \% \pm 4 \%$.

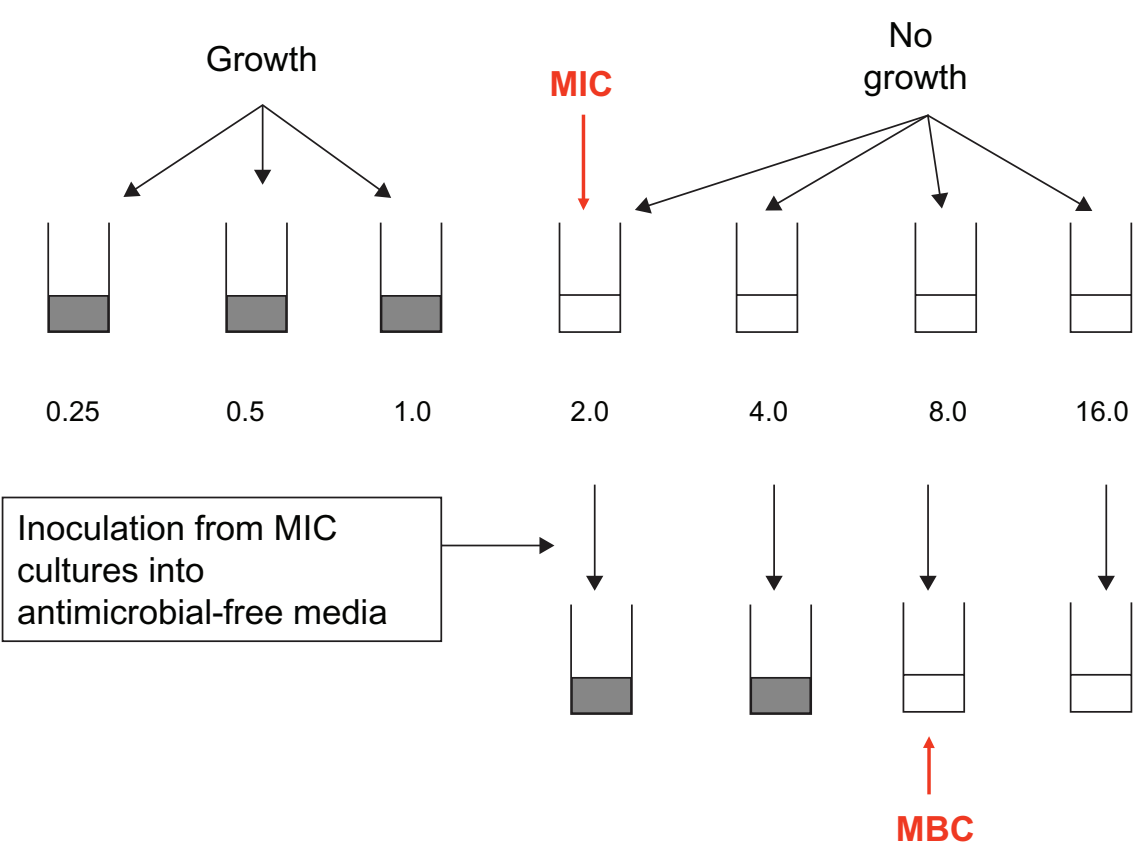

Figure I Design of serial dilution susceptibility testing method. 


\section{Drug release profiles and antimicrobial activity}

The release profile of amikacin from SLNs dispersion is shown in Figure 2a and 2b. Figure 2a shows the percentage of the drug released from the cholesterol carrier which was sustained for 70,144 , and $240 \mathrm{~h}$ at $40^{\circ} \mathrm{C}, 25^{\circ} \mathrm{C}$, and $4^{\circ} \mathrm{C}$, respectively. The differences between different temperatures before lyophilization are quite statistically significant (Figure 2a), but after lyophilization the differences are only significant between $4^{\circ} \mathrm{C}$ and $40^{\circ} \mathrm{C}$, and release was accelerated by increasing the storage temperature from $4^{\circ} \mathrm{C}$ to $40^{\circ} \mathrm{C}$ (Figure 2b). Desired freeze-dried SLNs were stored at $4^{\circ} \mathrm{C}$, $25^{\circ} \mathrm{C}$, and $40^{\circ} \mathrm{C}$, and drug release studies were done on each sample. Results show that after freeze drying only an early time burst release could be observed. By increasing the storage temperature, drug release was also accelerated.

The antimicrobial activity of SLNs of amikacin after preparation in first dispersion is shown in Figure 3a and antimicrobial activity of lyophilized particles after redispersion is shown in Figure 3b. It can be seen that SLNs of amikacin possessed antimicrobial activity against $P$. aeruginosa. Although the antimicrobial activity of drug-loaded SLNs was less than that of free drug (Table 1), it should be considered as it is shown in Figure 2a, that at $25^{\circ} \mathrm{C}$ after $24 \mathrm{~h}$ only about $12 \%$ of the loaded drug could be released from the carrier in vitro and this reached about $25 \%$ and $33 \%$ after 48 and $72 \mathrm{~h}$,
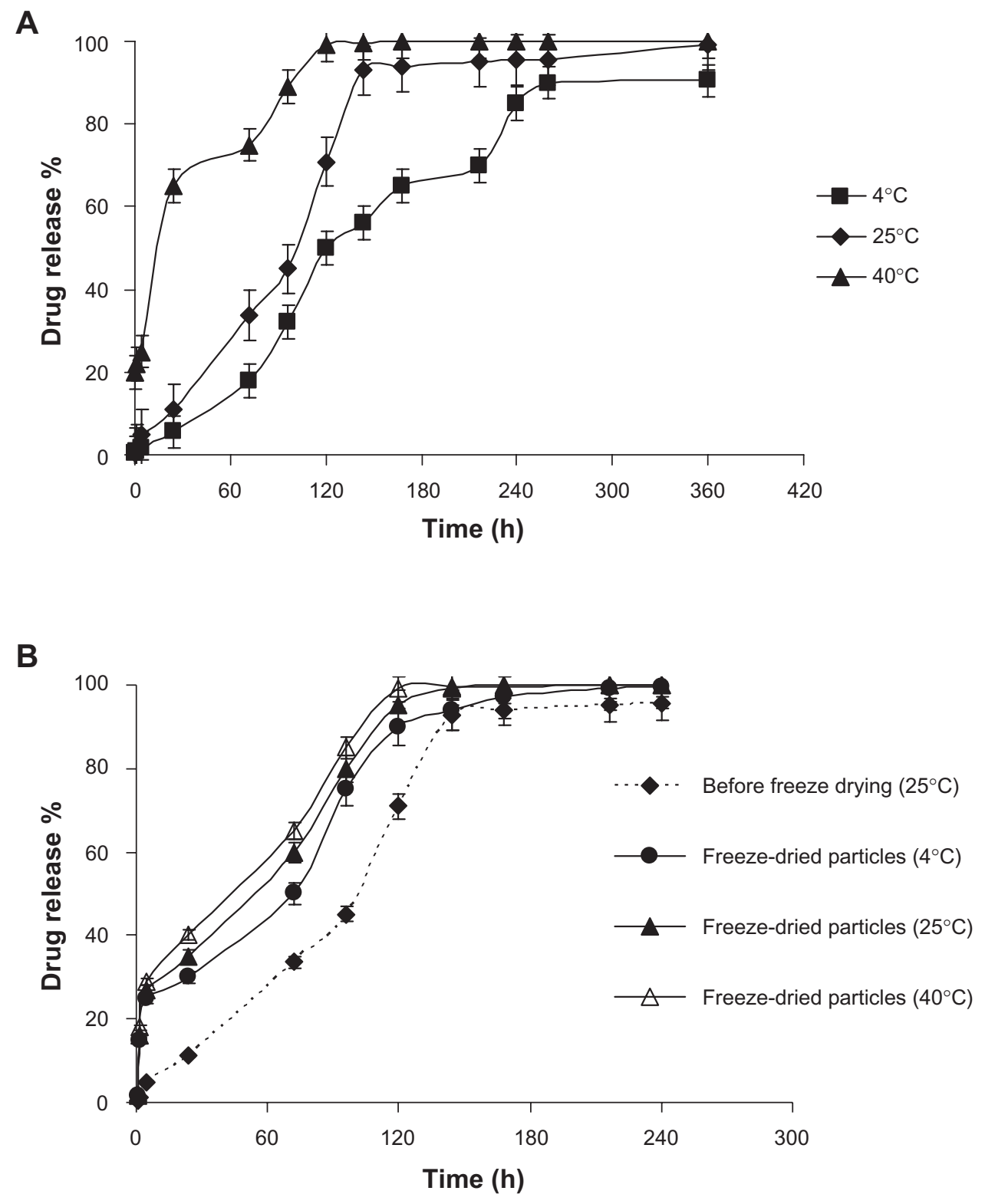

Figure 2 Release profiles of amikacin from SLNs at three different storage temperatures A) before and B) after freeze drying. 

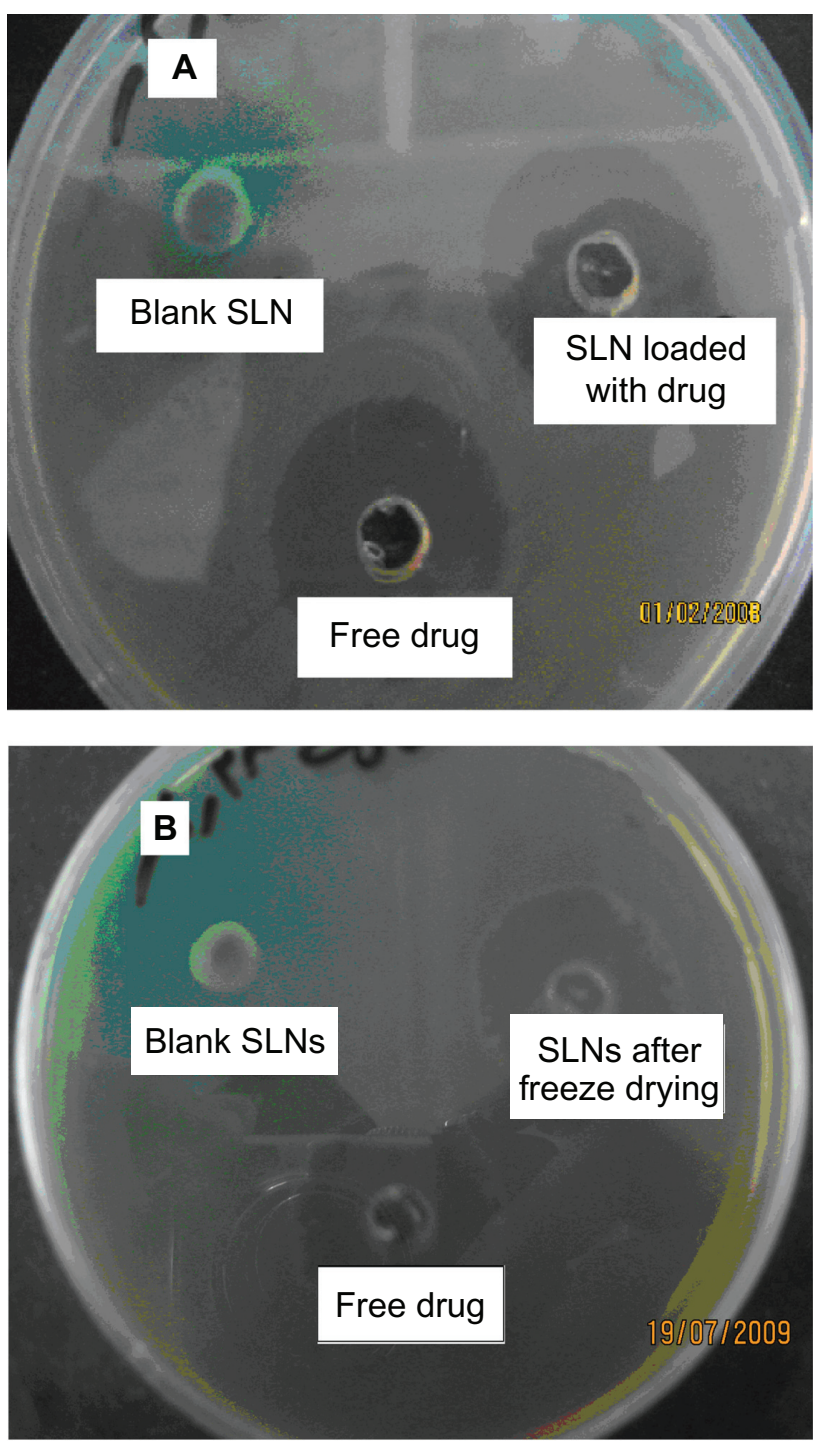

Figure 3 Photographs of the zone of inhibition produced by free amikacin and its SLNs in A) primary dispersion of SLNs and B) after freeze drying and re-dispersion of SLNs.

respectively. The percentage of drug release reaches to more than $95 \%$ of the loaded drug after about $144 \mathrm{~h}$. While in comparable time in which the antimicrobial effect was studied, in $48 \mathrm{~h}$ only $25 \%$ of amikacin was released from the SLNs dispersion. In this condition, the MIC and MBC of SLNs of amikacin was 16 and $8 \mu \mathrm{g} / \mathrm{mL}$ versus 8 and $4 \mu \mathrm{g} / \mathrm{mL}$ for free amikacin (Table 1) while the total concentration of released drug from SLNs of amikacin at this time is one fourth of the total concentration of free drug. Therefore, it could be estimated that the MIC and MBC of amikacin is half of the free drug in vivo after release of the entire loaded drug from the carrier. This more potent effect of SLNs of amikacin could be due to easier diffusion of lipid nanoparticles into the cellular membrane of $P$. aeruginosa cells as the lipid character of the cholesterol used in their production is similar to the cell wall of the bacteria. It may be concluded that this carrier could help with better transfer of aminoglycosides into the site of their action and possibly reduce the required dose and consequently the undesired side effects. Also small particle size of the desired SLNs may enhance diffusion of drug into the bacterial cell.

Previously, Beaulac et al, reported that the liposome formulation composed of dipalmitoylphosphatidylcholine and dimyristoylphosphatidylglycerol encapsulating tobramycin showed a considerable antibiotic effect at concentration below the MIC of the free antibiotic in vitro. ${ }^{13}$ They argued that the enhanced antimicrobial effect may be due to a fusion mechanism of liposome formulation with the bacteria cell wall. Other studies were carried out concerning intracellular bacteria; they reported the possibility of further improving liposomal drug efficacy toward infected cells. ${ }^{19,20}$ It seems that SLNs and liposomal structures are both composed of lipids and may be comparable.

\section{Freeze drying}

The optimum condition for lyophilization was evaluated for the type and percentage of cryoprotectant and freezing temperature, the best results achieved with $12 \% \mathrm{w} / \mathrm{w}$ of sucrose as cryoprotectant at $-80^{\circ} \mathrm{C}$. As the results are shown elsewhere, ${ }^{16}$ in this condition the increase in particle size was minimum, the release profile changes were minimum, and SEM pictures showed spherical particles similar prior to freeze drying. Also increasing the charge of particles caused more stability after redispersion. ${ }^{16}$

\section{Morphology study}

SEM pictures of amikacin loaded SLNs are shown in Figure $4 \mathrm{a}-4 \mathrm{~d}$. Figure $4 \mathrm{a}$, relates to the SLNs of amikacin which were stored at $4^{\circ} \mathrm{C}$ for 60 days, aggregation of particles was not seen. Figure $4 \mathrm{~b}$ and $4 \mathrm{c}$ shows SEM pictures of amikacin loaded nanoparticles stored at $25^{\circ} \mathrm{C}$ and $40^{\circ} \mathrm{C}$, respectively. Aggregation of particles and particle size enlargement were seen in these temperatures due to melting of cholesterol. Figure 4d shows SEM picture of lyophilized redispersed particles, this figure confirms that freeze drying doesn't have any significant impact on the shape and size of SLNs.

\section{Stability study}

Table 2 shows the stability of freeze-dried SLNs stored in different temperatures. Comparison of particle sizes before and after lyophilization showed that after freeze drying an 
Table I MBC and MIC of free amikacin and SLNs of amikacin before and after freeze drying with respect to Pseudomonas aeruginosa

\begin{tabular}{llll}
\hline Type of antibacterial activity & Free amikacin $(\mu \mathrm{g} / \mathrm{mL})$ & Amikacin-SLNs $(\mu \mathrm{g} / \mathrm{mL})$ & Before freeze drying \\
\cline { 2 - 4 } & & After freeze drying & 8 \\
\hline MBC & 4 & 8 & 16 \\
MIC & 8 & 16 & 16 \\
\hline
\end{tabular}

Abbreviations: MBC, minimum bacteriostatic concentration; MIC, minimum inhibitory concentrations; SLN, solid lipid nanoparticles.

initial particle, size enlargement could be seen $(190 \mathrm{~nm}$ after lyophilization versus $150 \mathrm{~nm}$ before), but storage of freezedried particles, at higher temperatures did not cause any significant particle size increase. However, when amikacin loaded SLNs were stored in dispersion form (without freeze drying process), increasing storage temperature caused a rapid particle size growth (Table 2 and Figure $4 a-4 c$ ). The zeta potential of SLNs after lyophilization was higher than nonlyophilized particles, almost all PDIs were less than 0.5 after freeze drying. Antimicrobial effects of amikacin did not change by lyophilization of particles and release profile of drug from freeze-dried particles was no different when particles were stored at different temperatures. In all conditions just a burst effect (20\%) was observed, but release profile of the prepared dispersion of SLNs of amikacin that was stored at different temperatures showed differences (Figure 2b). Stability studies confirmed that lyophilization could be a suitable method to increase stability of particles over time. As Table 2 shows, after freeze drying zeta potential of particles was increased and this could be the result of decreasing the risk of particles aggregation and enlargement after redispersion. Also, after freeze drying particle size did not increase as much as increasing MIC and MBC in comparison with nonfreezedried SLNs (Figure 3a and 3b). Acceleration of drug release via cholesterol at higher storage temperatures could be because of melting cholesterol at higher temperatures and disturbing the crystalline structure of lipid carrier by heating. After freeze drying the SLNs and storage of nanoparticles at different temperatures, redispersed particles did
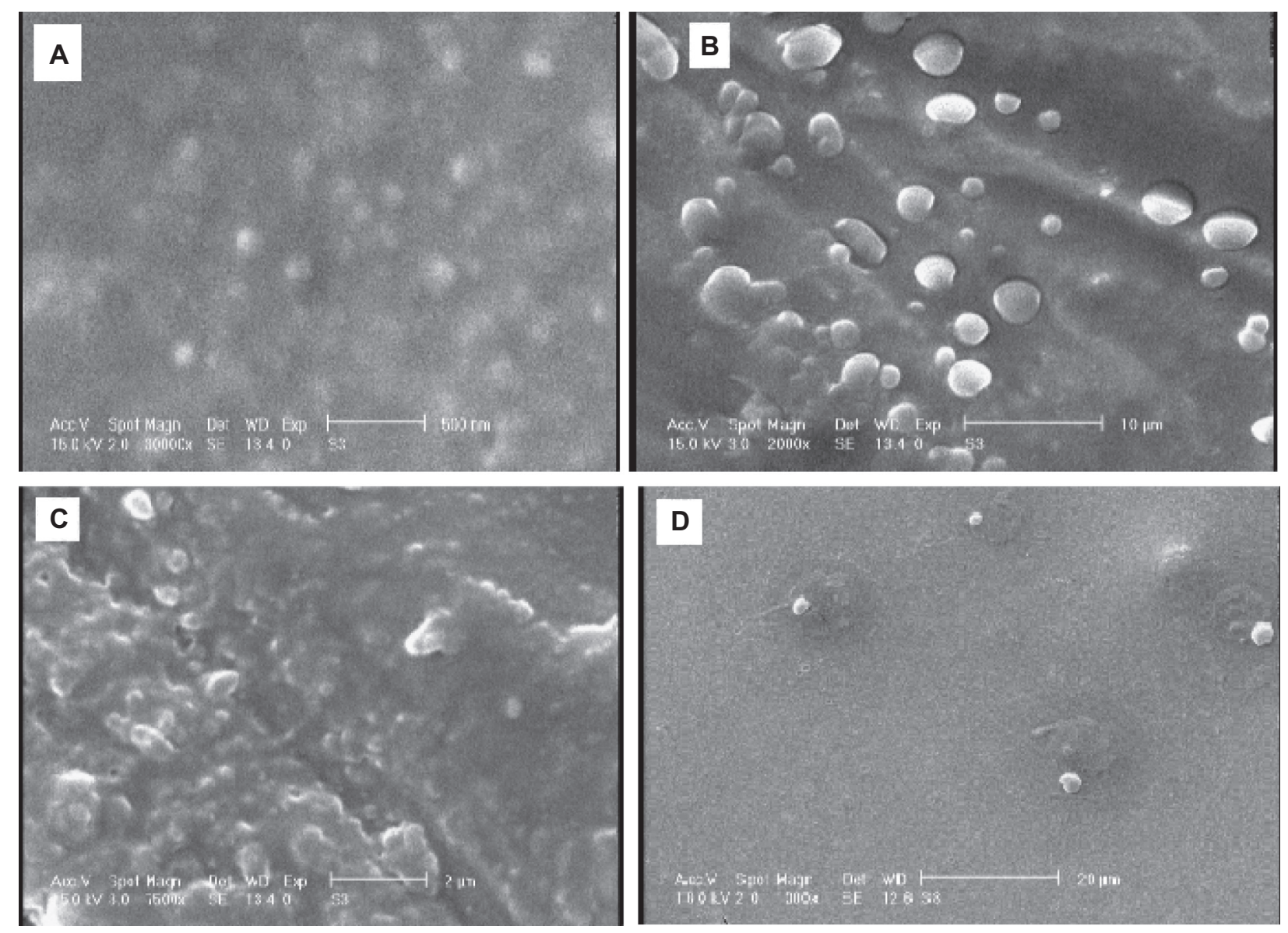

Figure 4 SEM photographs of SLNs of amikacin stored 60 days at A) $4^{\circ} \mathrm{C}$, B) $25^{\circ} \mathrm{C}$, C) $40^{\circ} \mathrm{C}$, and D) lyophilized SLNs stored at $40^{\circ} \mathrm{C}$. 
not show any difference when stored at different conditions $\left(4^{\circ} \mathrm{C}, 25^{\circ} \mathrm{C}\right.$, and $\left.40^{\circ} \mathrm{C}\right)$, and in all of them just some burst release was observed. Therefore, by lyophilization of desired SLNs of amikacin, stability of particles was increased without any significant changes in antimicrobial effects. Figure $4 \mathrm{a}-4 \mathrm{~d}$ confirms that freeze drying could be a suitable technique to reduce risk of particle size enlargement and particles aggregation.

\section{Conclusion}

The aim of this study was to evaluate the efficacy of the amikacin SLNs after preparation in the form of primary dispersion and redispersed freeze-dried particles in comparison with free drug. The loaded drug showed less MIC and MBC than free amikacin for both primary SLN dispersion and redispersed lyophilized particles. Some probable reasons were the lipophilic nature of SLNs which enhanced cellular entrance of drug into bacterial membrane and the small size of particles. Consequently it could be concluded that aminoglycosides might be administered in lower doses or longer intervals by delivering as solid lipid nanoparticles to reduce their side effects.

\section{Acknowledgments}

The authors would like to thank the Research Vice Chancellor of Isfahan University of Medical Sciences for financial support of this project. Technical assistance of Mr Jamali, Mrs S Norouzi, Mrs H Saabani, and Mr R Jafarian is appreciated. Thanks are given to Mrs Yousefi for English editing of the manuscript.

\section{References}

1. Bartal C, Danon A, Schlaeffer F, et al. Pharmacokinetic dosing of aminoglycosides: a controlled trial. Am J Med. 2003;114(3):194-198.

2. Schiffelers R, Storm G, Bakker-Woudenberg I. Liposome-encapsulated aminoglycosides in pre-clinical and clinical studies. $J$ Antimicrob Chemother. 2001;48(3):333-344.

3. Wilson JF, Davis AC, Tobin CM. Evaluation of commercial assays for vancomycin and aminoglycosides in serum: a comparison of accuracy and precision based on external quality assessment. J Antimicrob Chemother. 2003;52(1):78-82.

4. Hammond LJ, Galdwell S, Campbell PW. Cystic fibrosis, intravenous antibiotics, and home therapy. J Pediatr Health Care. 1991;5(1): 24-30.

5. Shah S, Misra A. Liposomal amikacin dry powder inhaler: effect of fines on in vitro performance. AAPS Pharm Sci Tech. 2004;5(4):e65.

6. Cullen AB, Cox CA, Hipp SJ, Wolfson MR, Shaffer TH. Intra-tracheal delivery strategy of gentamicin with partial liquid ventilation. Resp Med. 1999;93(11):770-778.

7. Atyabi F, Talaie F, Dinarvand R. Thiolated chitosan nanoparticles as an oral delivery system for amikacin: in vitro and ex vivo evaluations. $J$ Nanosci Nanotechnol. 2009;9(8):4593-4603.

8. Varshosaz J, Ghaffari S, Khoshayand MR, Atyabi F, Azarmi S, Kobarfard F. Development and optimization of amikacin solid lipid nanoparticles by central composite design. J Liposome Res. 2010;20(2): 97-104. 
9. Barani H, Montazer M. A review on applications of liposomes in textile processing. J Liposome Res. 2008;18(3):249-262.

10. Muller H, Mader K, Gohla S. Solid lipid nanoparticles (SLN) for controlled drug delivery - a review of the state of the art. Eur J Pharm Biopharm. 2000;50:161-177.

11. Zhang N, Ping Q, Huang G, Xu W, Cheng Y, Han X. Lecitin-modified solid lipid nanoparticles as carriers for oral administration of insulin. Int J Pharm. 2006;327(1-2):153-159.

12. Sweetman SC, editor. The Complete Drug Reference. 35 th ed. London, UK: Chicago Pharmaceutical Press (PhP); 2007.

13. Beaulac C, Sachetelli S, Lagace J. In vitro bactericidal efficacy of sub-MIC concentration of liposome encapsulated antibiotic against gram- negative and gram- positive bacteria. J Antimicrob Chemother. 1998;41(1):35-41.

14. Gazori T, Khoshayand MR, Azizi E, Yazdizade P, Nomani A, Haririan I Evalution of alginate/chitosan nanoparticles as antisense delivery vector: formulation, optimization, and in vitro characterization. Carbohyd Polym. 2009;77(3):599-606.

15. Varshosaz J, Ghaffari S, Khoshayand MR, Atyabi F. Optimization of freeze-drying condition of amikacin solid lipid nanoparticles using D-optimal experimental design. Pharm Dev Tech. 2010; In press.
16. Vivek K, Reddy H, Murthy R. Investigation of the effect of the lipid matrix on drug entrapment, in vitro release, and physical stability of olanzapine-loaded solid lipid nanoparticles. AAPS Pharm Sci Tech. 2007;8(4):16-24.

17. Larson TA, Peterson LR, Gerding DN. Microdilution aminoglycoside susceptibility testing of Pseudomonas aeruginosa and Escherichia coli: correlation between MICs of clinical isolates and quality control organisms. J Clin Microbiol. 1985;22(5):819-821.

18. Siddiqi S, Heifets LB, Cynamon M. Rapid broth macrodilution method for determination of MICs for Mycobacterium avium isolates. J Clin Microbiol. 1993;31(9):2332-2338.

19. Bermudez LE, Wu M, Young LS. Intracellular killing of Mycobacterium avium complex by rifapentine and liposome-incapsulated amikacin. J Infect Dis. 1987;156(3):510-513.

20. Poyner EA, Alpar HO, Almeida AJ, Gamble MD, Brown MRW. A comparative study on the pulmonary delivery of tobramycin encapsulated into liposomes and PLA microspheres following intravenous and endotracheal delivery. J Control Rel. 1995;35(1):41-48.
International Journal of Nanomedicine

\section{Publish your work in this journal}

The International Journal of Nanomedicine is an international, peerreviewed journal focusing on the application of nanotechnology in diagnostics, therapeutics, and drug delivery systems throughout the biomedical field. This journal is indexed on PubMed Central, MedLine, CAS, SciSearch $®$, Current Contents $\AA /$ Clinical Medicine,

\section{Dovepress}

Journal Citation Reports/Science Edition, EMBase, Scopus and the Elsevier Bibliographic databases. The manuscript management system is completely online and includes a very quick and fair peer-review system, which is all easy to use. Visit http://www.dovepress.com/ testimonials.php to read real quotes from published authors. 